\title{
WAVE ATTENUATION MODELLING BY SUBMERGED VEGETATION: ECOLOGICAL AND ENGINEERING ANALYSIS
}

\author{
Maria Maza', Javier L. Lara, Barbara Ondiviela and Inigo J. Losada
}

\begin{abstract}
The correct address of wave characteristics in the vicinity of submerged vegetation is crucial to perform an ecological analysis. Although several attempts have been done in the past using an analytical approach or depth averaged models, the rigidity of the assumptions used to solve the physics produced limited application to real cases. The use of a NS model called IH-2VOF is used first to minimize the number of predefined assumptions for wave propagation and the non-linear interactions between waves and plants and second to explore the possibility to improve existing turbulence models to consider wave interaction with vegetation. The IH2-VOF model has been validated using large scale experiments developed by Stratigaki et al. (2011). The model has shown a high degree of accordance between the lab data and the numerical predictions in free surface evolution. Numerical predictions of the velocity field have been compared both over and inside the vegetation showing also a high degree of accordance. Drag coefficients obtained during the model calibration are in accordance with previous studies such as Mendez et al. (1999). The influence of wave height, wave period, water depth and patch density have been studied using additional numerical simulations with irregular waves. Both the wave period and the water depth have been revealed as the most important parameters in the modification of the flow patterns around the patch.
\end{abstract}

Keywords: Seagrass; Navier-Stokes modeling; Wave plant interaction; Drag coefficient

\section{INTRODUCTION}

During the present era of global change, sustainable coastal protection is of growing importance. Hence, knowledge about the mitigation of flooding and erosion hazards with low environmental impact structures is of great interest. Coastal vegetation like salt marshes and seagrasses can play an important role in dissipating energy from waves and currents. Moreover, these ecosystems provide a very high ecological and economical values, which in part are also related to their capacity to dissipate hydrodynamic energy. The growing awareness of the potential of salt marshes and seagrasses in contributing to coastal protection has lead to a growing number of experimental and modeling studies, to get a mechanistic understanding on how these vegetations interact with hydrodynamics. Submerged vegetation exists in areas where the biotic and abiotic conditions are within the specific tolerance levels. Important factors are the minimum and maximum water depths at which vegetation can be found, determined by wave orbital velocity, tide, and light and the maximum and minimum current velocities. Besides these factors others might be locally important that could limit vegetation occurrence, such as nutrient availability (mainly $\mathrm{N}$ or $\mathrm{P}$ ), toxic sulphide conditions, eutrophication, temperature, oxygen, or organic matter content (De Boer, 2007). The necessity of relate hydrodynamic and ecological parameters is revealed essential to understand the behavior the ecosystems.

The correct address of wave characteristics in the vicinity of submerged vegetation is crucial to perform an ecological analysis. Although several attempts have been done in the past using an analytical approach (Dalrymple et al., 1984; Kobayashi et al.; 1993) the rigidity of the assumptions used to solve the physics produced limited application to real cases. Contrary to terrestrial canopies where wind effect on plants has been broadly investigated numerically, only a few applications have been presented to study wave attenuation by vegetation. The combined effect of wave propagation and plant induced damping has conditioned the numerical approach. Initial attempts were centered on the use of potential flow theory (Asano et al.; 1993) focusing on the determination of vegetation induced drag on waves. Mendez et al. (1999) modelled wave height evolution, vegetation and fluid motion on the vegetation under irregular wave conditions, analyzing the consequences on wave transformation. Further analysis focuses on more sophisticated wave propagation models, such as Boussinesq equations (Augustin et al., 2009) have also shown a good performance. However, all those works were focused on the effect of vegetation on wave without paying attention to the flow characteristics within the vegetation field. Recent experimental approaches (i.e.: Luhar et al., 2010) have revealed that the wave induced orbital velocity within the canopy was not significantly damped, revealing a relevant vertical motion within the plant field, pointing out the necessity of considering such aspect in the modeling.

\footnotetext{
${ }^{1}$ Environmental Hydraulics Institute, Universidad de Cantabria, C/ Isabel Torres 15, Santander, Cantabria, 39011, Spain, mazame@unican.es
} 
In this work a new approach based on the use of a Navier-Stokes model is presented. The model is able to provide the velocity field distribution not only around the vegetation field but also inside the meadow. It is providing very useful information to later link flow parameters with ecological indicators to study aspects such as plants health, potentials of sediment accretion or nutrients transport.

The paper is organized as follows. First, a description of the numerical model is performed. The numerical model validation using experimental data is presented next. Numerical results studying the influence of wave parameters on the flow around a submerged vegetation patch are shown. Finally, some conclusions are drawn.

\section{NUMERICAL IMPLEMENTATION}

\section{Equations}

Navier-Stokes (NS) models (successfully applied on wave-structure interaction problems; Losada et al., 2008), are able to solve the complete velocity field and turbulent magnitudes. Although applications mainly using a two dimensional approach have been presented to submerged plants using depth averaged models (i.e.: Augustin et al., 2009), several aspects regarding the complete velocity field modeling are still open. From our point of view the use of a NS model would give us the possibility of first, to minimize the number of predefined assumptions for wave propagation and the non-linear interactions between waves and plants and second to explore the possibility to improve existing turbulence models to consider wave interaction with vegetation. The IH2-VOF model, presented by Lara et al. (2011) is used in this work. IH-2VOF solves wave flow in a coupled NS-type equation system. The movement of free surface is tracked by the Volume of Fluid (VOF) method. A k$\varepsilon$ model is also considered for turbulence. An additional drag force induced by the meadow on the flow is considered in IH2-VOF in order to incorporate the momentum damping produced by vegetation. Drag force is introduced as a function of the width of the plant, $b$, the number of plants per unit area, $N$, the drag coefficient, $C_{D}$ and the horizontal fluid velocity inside the meadow, $\mathrm{u}$, as follows:

$$
\begin{gathered}
\bar{F}_{D, i}=\frac{1}{2} \cdot \rho \cdot C_{D} \cdot a \cdot N \cdot \bar{u}_{i} \cdot\left|\bar{u}_{i}\right| \\
\frac{\partial \bar{u}_{i}}{\partial x_{i}}=0
\end{gathered}
$$

Turbulence is modelled using a k- $\varepsilon$ equation model for the turbulent kinetic energy $(k)$, and the turbulent dissipation rate $(\varepsilon)$ which takes into account the effect of vegetation (see equations 3 and 4 ). The influence of turbulence fluctuations on the mean flow field is represented by the Reynolds stresses. The governing equations for $\mathrm{k}-\varepsilon$ are derived from the Navier-Stokes equations, and higher order correlations of turbulence fluctuations in $\mathrm{k}$ and $\varepsilon$ equations are replaced by closure conditions. The effect of the vegetation field is considered by two additional terms, one in the turbulent kinetic budget, $k_{w}$, and the other one in the turbulent dissipation rate, $\varepsilon_{w}$. These terms take into account the production of turbulent kinetic energy and the energy dissipation produced inside the vegetation field by an approximation of dispersive fluxes using the drag force produce by leaves (Hiraoka et al., 2006).

$$
\frac{\partial \bar{u}_{i}}{\partial t}+\bar{u}_{j} \frac{\partial \bar{u}_{i}}{\partial x_{j}}=-\frac{1}{\rho} \frac{\partial \bar{p}}{\partial x_{i}}+g_{i}+\frac{1}{\rho} \frac{\partial \overline{\tau_{i j}}}{\partial x_{j}}-\frac{\partial\left(\overline{u_{i}^{\prime} u_{j}^{\prime}}\right)}{\partial x_{j}}-\bar{F}_{D, i}
$$




$$
\begin{aligned}
& \rho \frac{\partial k}{\partial t}+\rho \bar{u}_{j} \frac{\partial k}{\partial x_{j}}=\frac{\partial}{\partial x_{j}}\left[\left(\frac{\mu_{t}}{\sigma_{k}}+\mu\right) \frac{\partial k}{\partial x_{j}}\right]+\tau_{i j} \frac{\partial \bar{u}_{i}}{\partial x_{j}}-\rho \varepsilon+ \\
& +\underbrace{\rho C_{k p} C_{D} a \sqrt{\bar{u}_{j} \bar{u}_{j}} k}_{k_{w}}
\end{aligned}
$$

$$
\begin{aligned}
& \rho \frac{\partial \varepsilon}{\partial t}+\rho \bar{u}_{j} \frac{\partial \varepsilon}{\partial x_{j}}=\frac{\partial}{\partial x_{j}}\left[\left(\frac{\mu_{t}}{\sigma_{\varepsilon}}+\mu\right) \frac{\partial \varepsilon}{\partial x_{j}}\right]+C_{\varepsilon 1} \frac{\varepsilon}{k} \tau_{i j} \frac{\partial \bar{u}_{i}}{\partial x_{j}}-C_{\varepsilon 2} \rho \frac{\varepsilon^{2}}{k}+ \\
& +\underbrace{\rho C_{\varepsilon p} C_{D} a \sqrt{\bar{u}_{j} \bar{u}_{j}} \varepsilon}_{\varepsilon_{w}}
\end{aligned}
$$

\section{Validation}

Although an extensively validation of IH-2VOF model have been done, validation for this new implementation is needed. The vegetation effect in the flow is validated using Stratigaki et al. (2011) experiments. This effect is introduced considering the drag force presented in equation (1) obtained as a function of the flow velocity (Méndez et al., 1999; Lowe et al., 2005). The only parameter considered during the calibration is drag coefficient, $\mathrm{C}_{\mathrm{D}}$, keeping constant the turbulence model parameters.

Stratigaki et al. (2011) experiments were carried out in the CIEM (Canal d'Investigatió i Experimentació Marítima) flume in Barcelona. Experiments were performed scale at the 100m long, $3 \mathrm{~m}$ wide and $5 \mathrm{~m}$ deep flume. A $0.7 \mathrm{~m}$ thick and $12 \mathrm{~m}$ long sand layer was disposed to cover the flume bottom. The sandy bed was setup at the bottom of the wave flume. First a 1/15 sloped bed was located starting $17.21 \mathrm{~m}$ from the wave-maker. A $20 \mathrm{~m}$ long horizontal bottom region was disposed. The vegetation mimics were installed in this region starting $38.36 \mathrm{~m}$ from the wave paddle. Finally a $37 \mathrm{~m}$ long beach was built to dissipate wave energy.

Vegetation mimics were composed of two $45 \mathrm{~cm}$ long PVC strips and another pair of $27.5 \mathrm{~cm}$ length, all inserted in a stiff $10 \mathrm{~cm}$ long rod of the same material. PVC strips were $1 \mathrm{~mm}$ thick and $1 \mathrm{~cm}$ wide, with a Young's modulus equal to $2.9 \mathrm{GPa}$ and a density equal to $700 \mathrm{~kg} / \mathrm{m} 3$.

Two densities were tested: one dense configuration with $360 \mathrm{mimics} / \mathrm{m}^{2}$ and one sparse configuration with $180 \mathrm{mimics} / \mathrm{m}^{2}$. Fifteen resistive gauges, six before the meadow, seven over it and two after the field were used to measured the free surface. Eight ADVs (Acoustic Doppler Velocimeters) and four EMCMs (Electro Magnetic Current-meters) were disposed in order to measure velocity. These devices were positioned at three different distances from the paddle, at 37.66, 40.36 and $46.36 \mathrm{~m}$ and four vertical positions. EMCMs allowed measuring velocity inside the field.

Numerical experiments were reproduced using the whole wave flume. The discretization used in this problem was $0.04 \mathrm{~m}$ in the horizontal dimension and $0.02 \mathrm{~m}$ in the vertical one. Four cases were chosen among the tested in the flume to calibrate the drag coefficient and to evaluate the performance of the model in reproducing the wave field. The tested wave conditions were $0.4 \mathrm{~m}$ and $0.5 \mathrm{~m}$ regular wave trains with 3, 3.5 and 4 s of wave period. Three water depths at the meadow location were used: 1.8, 2.0 and $2.4 \mathrm{~m}$. Two different densities were considered, 180 and $360 \mathrm{mimics} / \mathrm{m}^{2}$.

Figure 1 shows the wave height evolution along the field obtained for the five different cases used for the drag coefficient calibration. Numerical results are shown using a solid black line and laboratory measurements using black dots. Wave conditions, water depth and vegetation density are displayed. The obtained calibrated drag coefficient is also displayed.

Obtained results of the calibrated drag coefficient were found to be in agreement with the ones predicted by Mendez et al. (1999) when plant swaying is not included in the physics of the modeling. A more detailed analysis will be performed in the future in order to analyze the influence of the different numerical modeling approach or the effect of the coupled motion of the plant done to the wave induced velocities. Mendez et al. (1999) used a potential flow model and in this case a Reynolds Averaged Navier-Stokes model is used. 

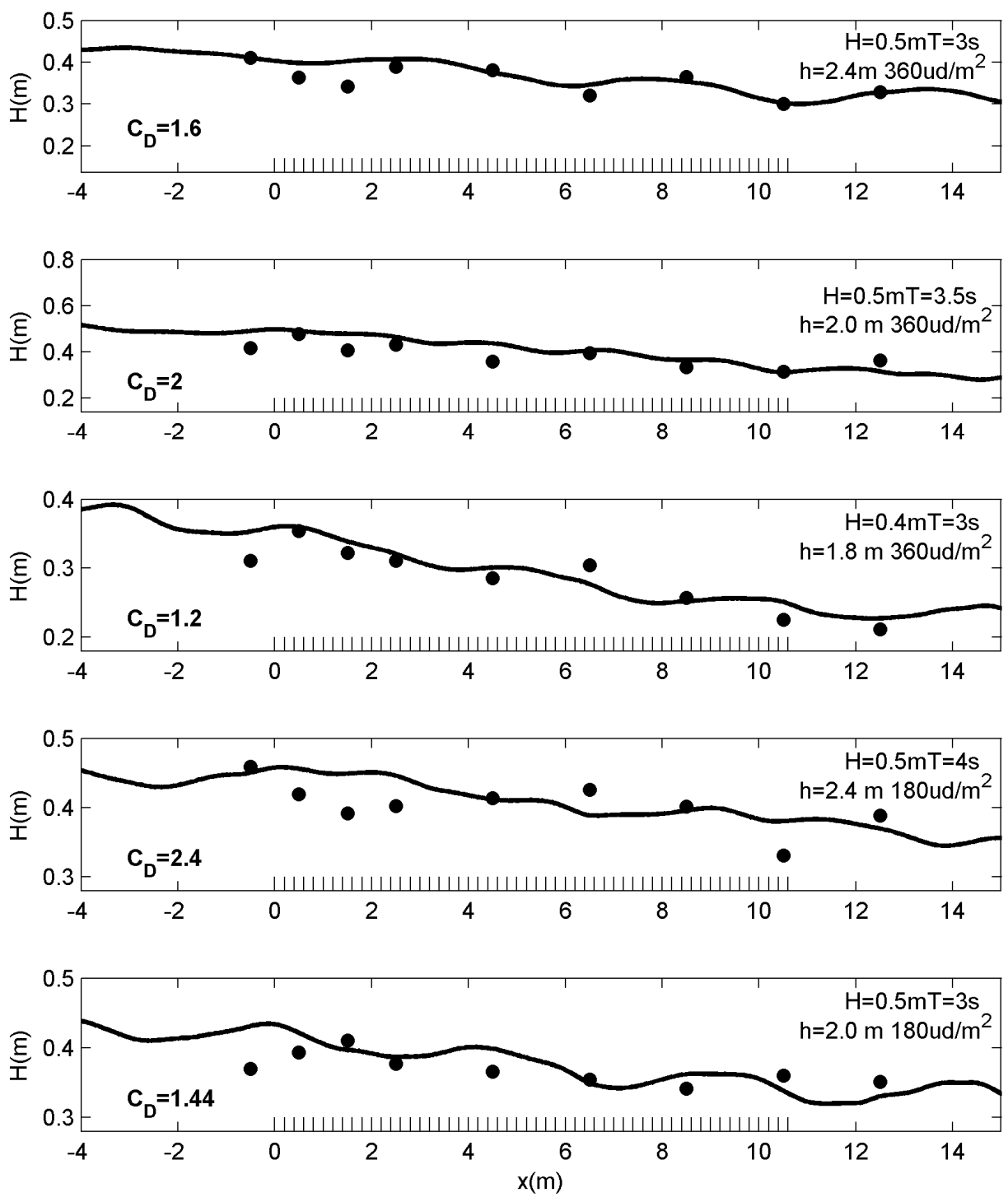

Figure 1. Wave evolution validation using Stratigaki et al. (2011) experiments. Black solid line represents the numerical results and black dots the laboratory measurements. Wave height $(H)$, wave period $(T)$, water depth $(h)$ and density $\left(\mathrm{ud} / \mathrm{m}^{2}\right)$ are displayed as well as the drag coefficient $\left(\mathrm{C}_{\mathrm{D}}\right)$.

As it can be seen in Figure 1 there is a good agreement between numerical and laboratory measurements for different wave conditions and meadow densities. Therefore, the model allows reproducing the wave attenuation along the vegetation meadow for different flow and vegetation characteristics.

IH-2VOF model allows obtaining velocities in the entire water column. Therefore, velocity measurements taken from Stratigaki et al. (2011) experiments are compared with the obtained numerical results. In order to analyze velocity changes along the vertical direction, velocity profiles are compared. They are obtained after averaging the horizontal velocity field over 40 waves. Velocity profiles at 37.66, 40.36 and $46.36 \mathrm{~m}$ from the paddle are represented and they are compared with the four laboratory measurements taken along the vertical in each one of those positions. Figure 2 shows the comparison between the numerical simulations (solid line) and the laboratory measurements (dots) for a $0.5 \mathrm{~m}$ high and a $3.5 \mathrm{~s}$ period regular wave. The water depth at the generation region is $2.4 \mathrm{~m}$ in this case. The density of the meadow is $180 \mathrm{mimics} / \mathrm{m}^{2}$. 

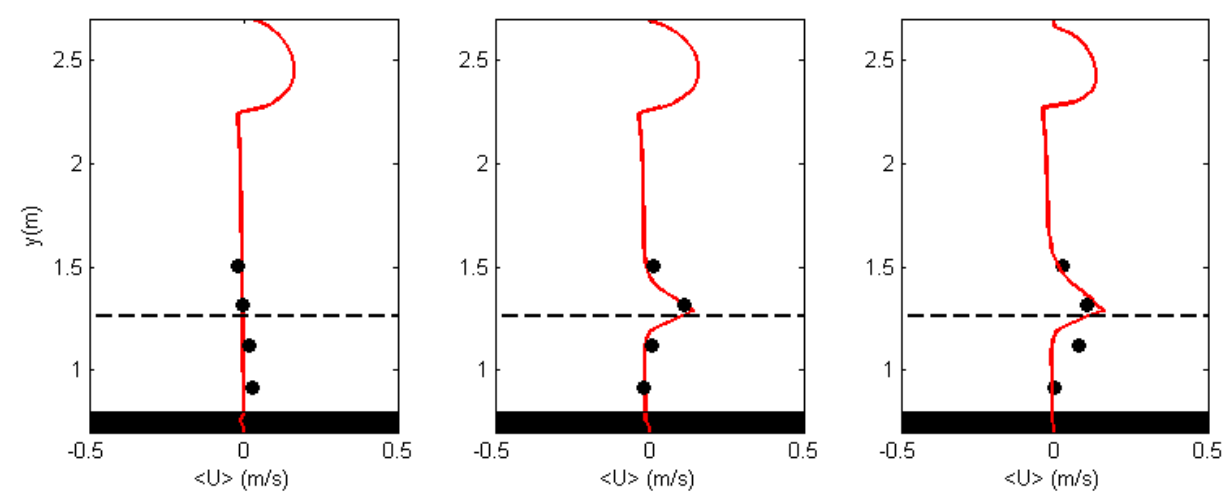

Figure 2. Horizontal velocity profiles validation using Stratigaki et al. (2011) experiments. Red solid line represents numerical results and black dots laboratory measurements. Horizontal dashed line represents the end of the vegetation field.

As it can be observed from the figure, there is a good agreement between laboratory and numerical data, reproducing with high precision the mean velocity values. The skimming flow produced above the vegetation field is reproduced by the model as well as the velocity reduction inside the meadow. Therefore, the model allows reproducing accurately the horizontal velocities inside and outside the meadow. A dramatic velocity reduction within the vegetation patch is also observed as expected. A large velocity gradient can be clearly detected at the upper part of the vegetation patch.

\section{NUMERICAL SIMULATIONS}

Once the model is validated some new numerical simulations are carried out in order to study the influence of different parameters in the loss of energy produced by submerged vegetation. An analysis in the wave flow is carried out. Different wave conditions are tested considering irregular wave conditions and different water depths. Significant wave height of 0.3 and 0.4 m with a 2 and 4 s peak period are considered. Wave energy is considered to be distributed following a Jonswap spectrum with a 3.3 peak enhancement factor. One hundred fifty waves are considered in the simulations to ensure statistical reliability of the results. The same values to those considered during the experiments are tested: 1.8 and $2.4 \mathrm{~m}$ of water depth and meadow density of 180 and $360 \mathrm{mimics} / \mathrm{m}^{2}$. The height and the plant characteristics are the same as the ones used in Stratigaki et al. (2011) experiments. The wave flume considered in the simulations is the same as the one used for the model validation. All the numerical simulations were performed with active wave absorption at the wave generation boundary in order to absorb reflected waves at the vegetation patch. Drag coefficient used in the simulations was obtained using Mendez et al. (1999) proposed formulation. Because that formulation was obtained for regular wave trains, the significant wave height and the mean period was chosen as the representative wave parameters to obtain the Reynolds number need in the formulation.

In order to study the influence of wave height, wave period, water depth and vegetation density in the energy dissipation the mean velocity profiles and the mean velocity fields are analyzed and discussed next.

\section{Non-dimensional velocity profiles}

Horizontal velocity profiles are studied in this section. Root mean square velocity values evaluated over 150 waves are considered. Velocity is normalized with the maximum velocity produced at the free end of the vegetation at the beginning of the vegetation field in order to directly relate that value with the Reynolds number used in the prediction of the drag coefficient. Four locations are chosen to analyze velocity profiles. The first profile is $0.06 \mathrm{~L}$ ( $\mathrm{L}$ is the length of the meadow, $10.7 \mathrm{~m}$ ) offshore the meadow. Two profiles are inside the meadow at $0.19 \mathrm{~L}$ and $0.75 \mathrm{~L}$. Finally, a profile is located $0.07 \mathrm{~L}$ onshore the meadow (denoted 1.07 in the figures).

The influence of wave peak period is analyzed considering two wave conditions, 2 and $4 \mathrm{~s}$ with the same significant wave height, $0.4 \mathrm{~m}$. The water depth and the vegetation density are also kept constant to $2.4 \mathrm{~m}$ and $180 \mathrm{mimics} / \mathrm{m}^{2}$, respectively. Figure 3 shows the velocity profiles obtained for those conditions. Vertical coordinate, $\mathrm{z}$, is normalized with water depth, h. 

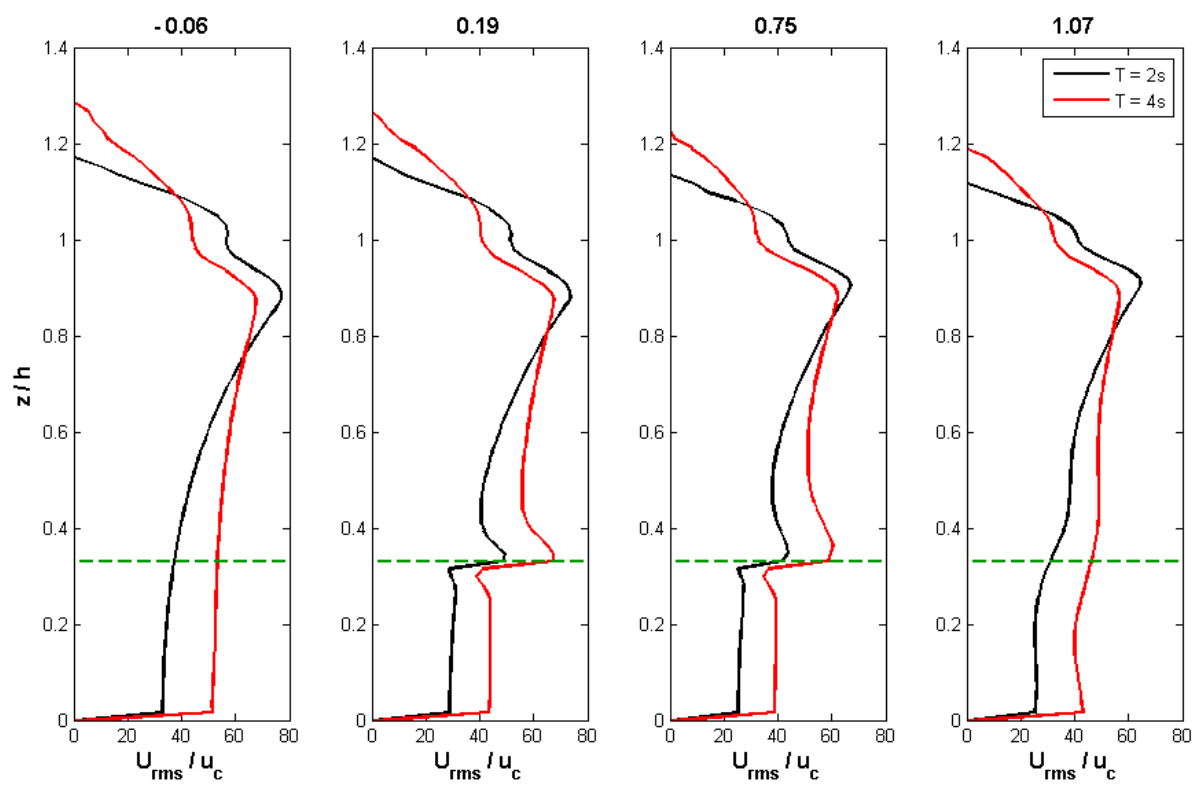

Figure 3. Comparison between two different wave conditions with the same wave height, $0.4 \mathrm{~m}$, significant water depth, $2.4 \mathrm{~m}$ and vegetation density, $180 \mathrm{mimics} / \mathrm{m} 2$, and two different wave peak periods, 2 and $4 \mathrm{~s}$. Black solid line represents $2 \mathrm{~s}$ wave period wave and red solid line $4 \mathrm{~s}$ wave period wave.

As it can be observed in Figure 3, a velocity reduction inside the field occurs in both cases. The skimming flow is only produced inside the meadow and the larger wave period seems to develop a larger non-dimensional velocity value. The larger Reynolds number created by the larger period wave train seems to be more effective in the generation of an increment in the velocity field over the vegetation. This larger velocity appears also to be more effective in the transmission of momentum inside the patch, as can be seen in the figure.

The same comparison is done, but in this case considering two different significant wave heights, $0.3 \mathrm{~m}$ and $0.4 \mathrm{~m}$. The rest of the parameters do not vary with respect to the previous case. Figure 4 shows the comparison of two simulations.
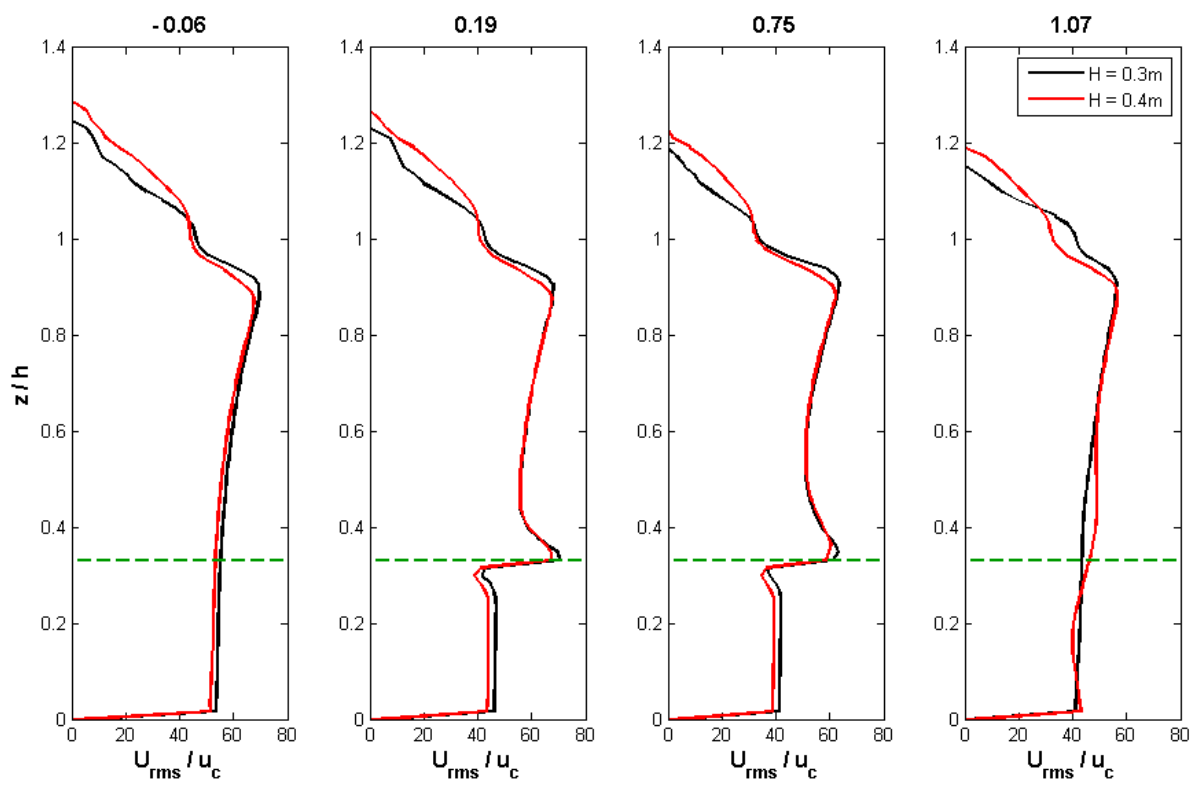

Figure 4. Comparison between two different wave conditions with the same wave peak period, $4 \mathrm{~s}$, significant water depth, $2.4 \mathrm{~m}$ and vegetation density, $180 \mathrm{mimics} / \mathrm{m} 2$, and two different wave heights, 0.3 and $0.4 \mathrm{~m}$. Black solid line represents the smallest wave height and red solid line the biggest one.

Figure 4 reveals a small influence of the wave height in the velocities developed inside and around the meadow. There is a slightly higher velocity reduction inside the meadow with higher wave height 
but the difference is not very significant. Therefore, the wave height has a smaller influence in the energy reduction produced by the meadow than the wave period. The transmission of momentum inside the meadow seems also to be not very dependent on the wave height.

The influence of the water depth is studied next. As in the previous cases two simulations are performed for water depth equal to 1.8 and $2.4 \mathrm{~m}$. The rest of parameters do not change. In this case, simulations are performed for a $0.3 \mathrm{~m}$ significant wave height and a 4 s peak period wave. Results are displayed in Figure 5.
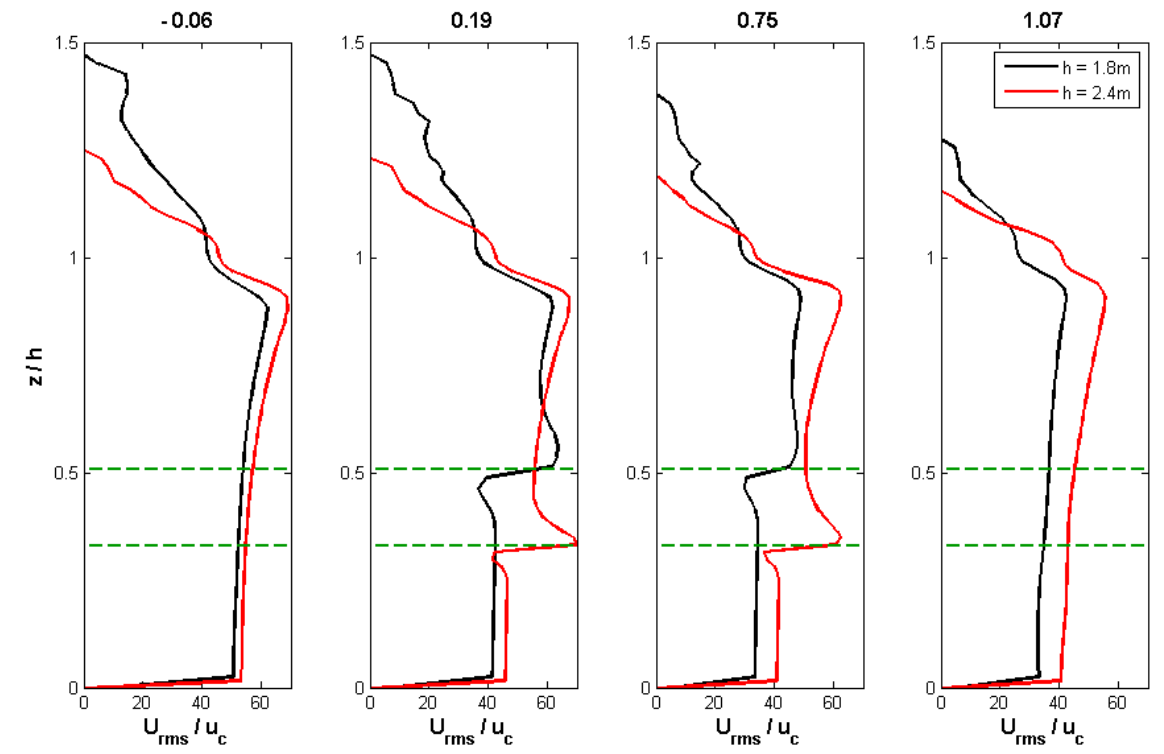

Figure 5. Comparison between two different wave conditions with the same wave peak period, $4 \mathrm{~s}$, significant wave height, $0.3 \mathrm{~m}$ and vegetation density, $180 \mathrm{mimics} / \mathrm{m} 2$, and two different water depths, 1.8 and $2.4 \mathrm{~m}$. Black solid line represents the smallest water depth and red solid line the biggest one.

Figure 5 shows two similar velocity profiles in shape. However, when the flow starts to enter in the vegetation field the velocity profile associated to the smallest water depth suffers a large reduction. This aspect is observed in the last image where the velocity reduction is higher for the smallest water depth.
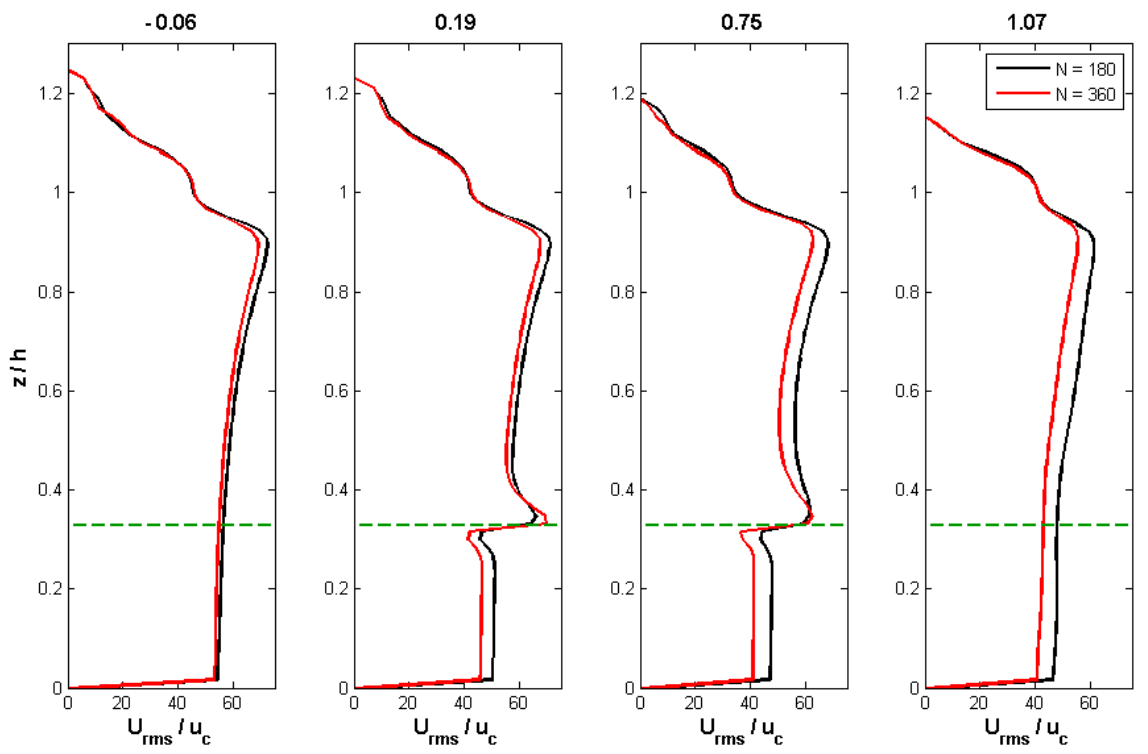

Figure 6. Comparison between two different vegetation densities, 180 and $360 \mathrm{mimics} / \mathrm{m}^{2}$, with the same wave peak period, $4 \mathrm{~s}$, significant wave height, $0.3 \mathrm{~m}$ and water depth, $2.4 \mathrm{~m}$. Black solid line represents the smallest vegetation density and red solid line the biggest one. 
The last studied variable is the vegetation density. Two simulations considering 180 and 360 mimics $/ \mathrm{m}^{2}$ are performed and the results are displayed in Figure 6, for a $0.3 \mathrm{~m}$ significant wave height and a $2.4 \mathrm{~s}$ peak period wave.

Figure 6 reveals the direct relationship between the vegetation density and the velocity reduction induced by the meadow. Dense meadows produce a higher reduction in flow velocities than sparse meadows. The vegetation density influence in the skimming flow is very important; it is the main factor which determines the development of this effect. This aspect is observed in Figure 6 where the skimming flow is larger for the denser configuration.

\section{Mean velocity fields}

The mean velocity fields around the vegetation are studied next. Because the numerical model is able to provide the information of the two-dimensional velocity field, a detailed analysis of the mean flow pattern produced around the vegetations is carried out.

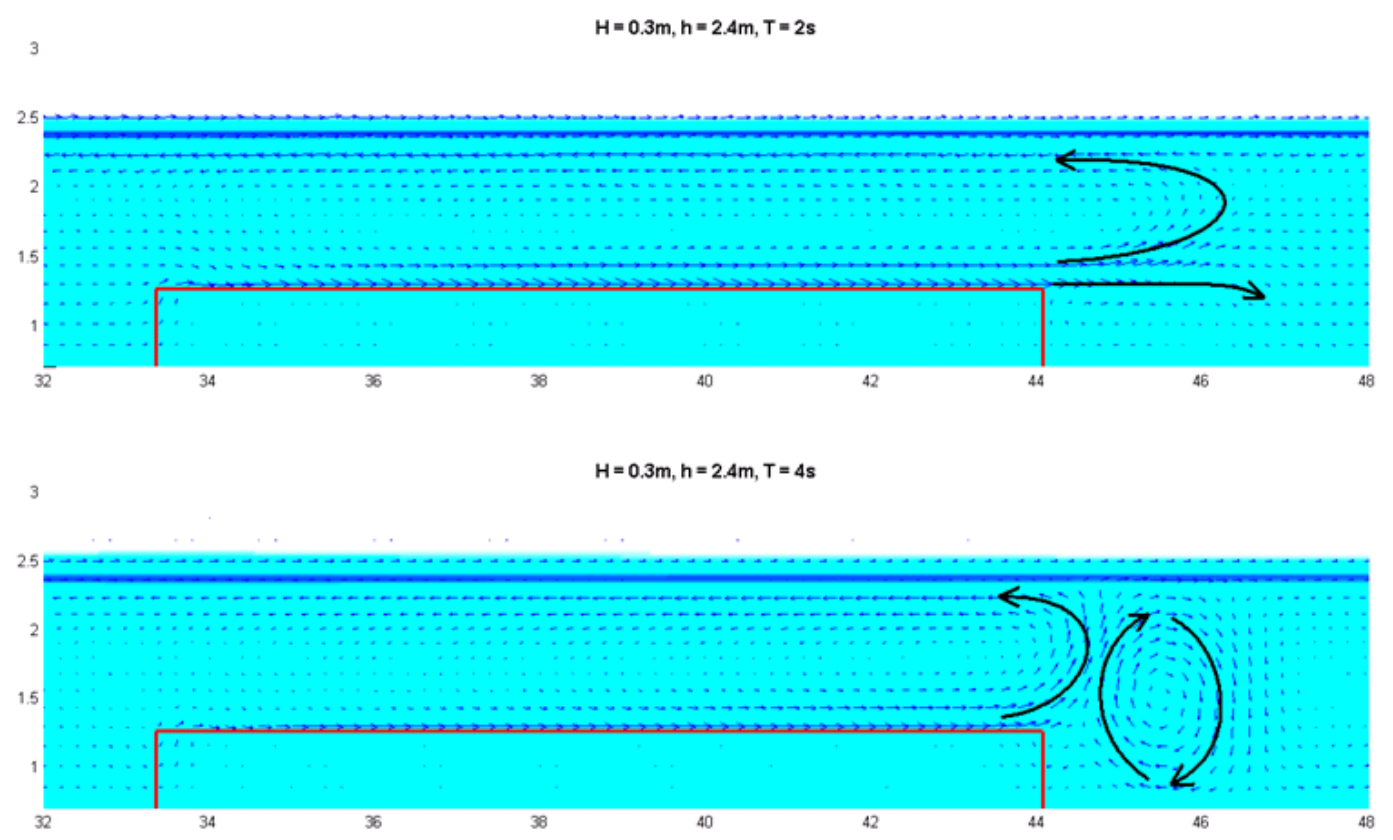

Figure 7. Mean velocity fields for two different wave conditions with the same significant wave height, $0.3 \mathrm{~m}$, water depth, $2.4 \mathrm{~m}$ and vegetation density, $180 \mathrm{mimics} / \mathrm{m}^{2}$, and two different wave peak periods, $2 \mathrm{~s}$ (above) and $4 \mathrm{~s}$ (below).

Figure 7 shows the influence of the peak period on the mean flow around the meadow. In this case a $0.3 \mathrm{~m}$ significant wave height is considered. The water depth is $2.4 \mathrm{~m}$ and the meadow density is 180 mimics $/ \mathrm{m}^{2}$. The upper panel shows the case for a $2 \mathrm{~s}$ peak wave period and the lower the $4 \mathrm{~s}$ peak period wave case. The location of the meadow is plotted using a red line.

As it was shown in the horizontal profile analysis, the influence of the wave period is evident. The same flow pattern is observed over the meadow. A skimming flow is developed as a consequence of the drag forced exerted by the plants. However, onshore the meadow, different recirculation patters are obtained from the simulations. Although an upwelling motion is observed in both panels at the rear end point of the vegetation patch, a large vortex occupying the whole water depth is only observed in the larger period wave case. The shorter period seems to develop a weak vortex onshore the patch with the same height than the plants. The existence of that vortex demonstrates the perturbation of the wave field due to the vegetation and the potential to develop an accretion of sediment onshore the meadow. This effect is demonstrated to be enhanced with the wave period. 


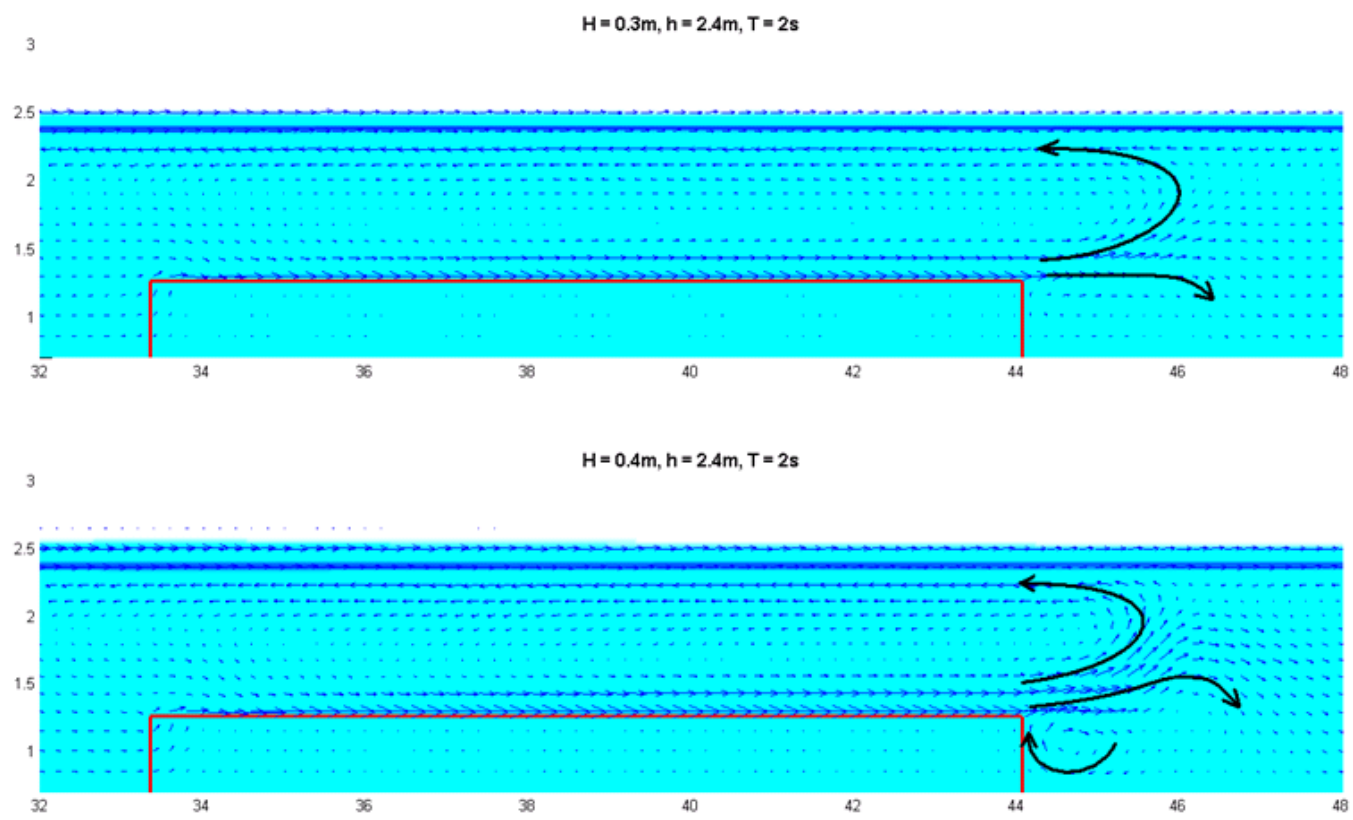

Figure 8. Mean velocity fields for two different wave conditions with the same wave peak period, $2 \mathrm{~s}$, water depth, $2.4 \mathrm{~m}$ and vegetation density, $180 \mathrm{mimics} / \mathrm{m}^{2}$, and two different wave heights, $0.3 \mathrm{~m}$ (above) and $0.4 \mathrm{~m}$ (below).

Figure 8 shows the numerical results for two cases with different significant wave height, 0.3 (upper panel) and $0.4 \mathrm{~m}$ (lower panel). Details about the wave characteristics are the same as the one used for the velocity profiles analysis. As it can be seen in the figure, in both situations the flow pattern is almost identical. Larger flow perturbation is observed for the largest wave height case at the onshore locations caused by the existence of a stronger current as a result of the skimming flow. This also induce a larger vortex behind the patch, but no large differences are found in both panels revealing a small influence of the wave height on the flow behavior around the meadow.
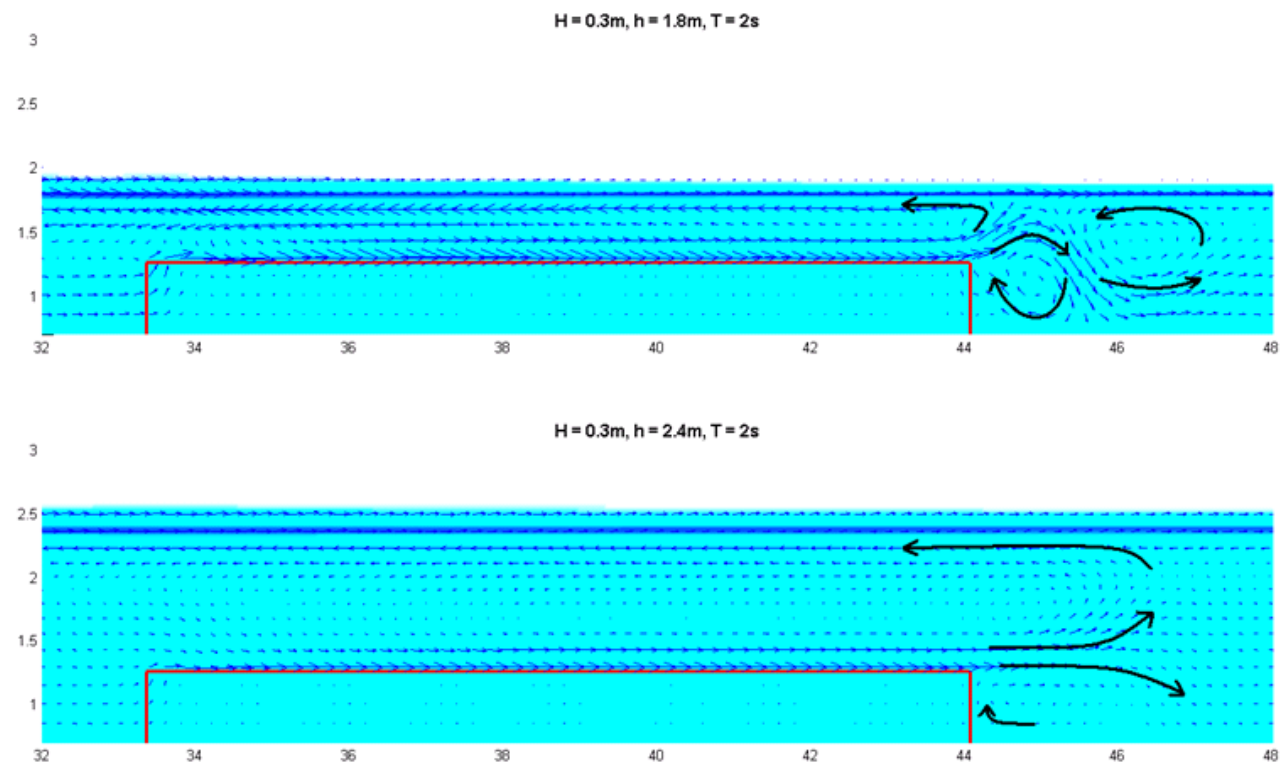

Figure 9. Mean velocity fields for two different wave conditions with the same wave height, $0.3 \mathrm{~m}$, wave peak period, $2 \mathrm{~s}$ and vegetation density, $180 \mathrm{mimics} / \mathrm{m}^{2}$, and two different water depths, $1.8 \mathrm{~m}$ (above) and $2.4 \mathrm{~m}$ (below). 
Finally, the effect of the water depth is investigated in figure 9 . In both cases a $0.3 \mathrm{~m}$ significant wave height and a $2 \mathrm{~s}$ peak period wave train is used. The upper panel corresponds to a $1.8 \mathrm{~m}$ water depth and the lower one corresponds to a $2.4 \mathrm{~m}$ water depth. The effect of the water depth is really significant, especially at the end of the meadow. The deepest water condition shows a more confined flow. The shallowest case seems to be more affected by the presence of the free surface. At the end of the vegetation, the skimming flow projects the jet produced over the plants upwards, inducing a vortex close to the bottom. This effect can also be related with the potential of sediment transport to be accumulated after the meadow. The effect of the water depth seems to be relevant in the flow induced patterns as described by Lowe et al. (2005).

\section{CONCLUSIONS}

The correct address of wave characteristics in the vicinity of submerged vegetation is crucial to perform an ecological analysis. Although several attempts have been made in the past using an analytical approach or depth averaged models, the rigidity of the assumptions used to solve the physics produce limited application to real cases. The use of a NS model called IH-2VOF is used first to minimize the number of predefined assumptions for wave propagation and the non-linear interactions between waves and plants and second to explore the possibility to improve existing turbulence models to consider wave interaction with vegetation. The IH-2VOF model has been validated using large scale experiments carried out by Stratigaki et al. (2011). The model has shown a high degree of accordance between the laboratory data and the numerical predictions in free surface evolution. Numerical predictions of the velocity field have been compared both over and inside the vegetation, showing also a high degree of accordance. Drag coefficients obtained during the model calibration are in accordance with previous studies such as Mendez et al. (1999). The influence of wave height, wave period, water depth and patch density have been studied using additional numerical simulations with irregular waves. Both the wave period and the water depth have been revealed as the most important parameters in the modification of the flow patterns around the patch.

Further analysis has to be performed in order to parameterize that effect and to later link to additional physical processes such as sediment and nutrients transport.

\section{REFERENCES}

Asano, T., Tsutsui, S., and Sakai, T. 1988. Wave damping characteristics due to seaweed, Procceedings of 25th Coastal Engineering Conference in Japan, 138-142.

Augustin, L.N., Irish, J.L., and Lynett, P. 2009. Laboratory and numerical studies of wave damping by emergent and near emergent wetland vegetation, Coastal Engineering, 56, 332-340.

Dalrymple, R.A., Kirby, J.T., and Hwang, P.A. 1984. Wave Diffraction Due to Areas of Energy Dissipation, Coastal and Ocean Engineering. J. Wtrwy., Port, Vol. 110, Issue 1, pp. 67-79.

De Boer, W.F. 2007. Seagrass-sediment interactions, positive feedbacks and critical thresholds for occurrence: a review. Hydrobiologia, 591: 5-24.

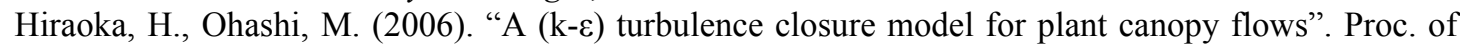
4th International Symposium on Computational Wind Engineering (CWE2006) in Yokohama.

Kobayashi, N., Raichlen, A.W., and Asano T. (1993). Wave Attenuation by Vegetation, Coastal and Ocean Engineering, J. Wtrwy., Port Vol. 119, 30-48.

Lara, J.L., Ruju, A., and Losada, I.J. 2011. Reynolds averaged Navier-Stokes modelling of long waves induced by a transient wave group on a beach, Proceedings of the royal society A. 467: 12151242.

Losada, I.J., Lara, J.L., Guanche, R., Gonzalez-Ondina, J.M. (2008). "Numerical analysis of wave overtopping of rubble mound breakwaters", Coastal Engineering, ELSEVIER. 55 (1), 47-62.

Lowe, R.J., Koseff, J.R., Monismith, S.G. (2005). "Oscillatory flow through submerged canopies. Part 1. Velocity structure", J. of Geophysical Research. 110, doi: 10.1029/2004JC002788.

Luhar, M., Coutu, S., Infantes, E., Fox, S. and Nepf, H. (2010). "Wave-induced velocities inside a model seagrass bed". J. of Geophysical Research, vol. 115, C12005.

Mendez F.J. and Losada I.J., (1999). "Hydrodynamics induced by wind waves in a vegetation field", $J$. of Geophysical Research, vol. 104, C8, 18383-18396.

Stratigaki, V., Manca, E., Prinos, P., Losada, I.J., Lara, J.L., Sclavo, M., Amos, C.L., Caceres, I., Sanchez-Arcilla, A. (2011). Large scale experiments on wave propagation over Posidonia Oceanica. Journal of Hydraulic Research, IAHR, Vol. 49, Supplement 1, 31-43. 